\title{
Research and Design of a Sample Heater for Beam Line 6-2c Transmission X-ray Microscope
}

\author{
Veronica Policht \\ Office of Science, Science Undergraduate Laboratory Internship (SULI) \\ Loyola University, Chicago \\ SLAC National Accelerator Laboratory \\ Stanford, CA
}

August 12, 2011

\begin{abstract}
Prepared in partial fulfillment of the requirements of the Office of Science, Department of Energy's Science Undergraduate Laboratory Internship under the direction of Johanna Nelson at the Stanford Synchrotron Radiation Lightsource, SLAC National Accelerator Laboratory.
\end{abstract}

Participant:

Signature

Research Advisor:

Signature 


\section{TABLE OF CONTENTS}

$\begin{array}{lc}\text { Abstract } & \text { ii } \\ \text { Introduction } & 1 \\ \text { Methods } & 5 \\ \text { Results } & 6 \\ \text { Conclusion } & 13 \\ \text { Acknowledgement } & 13 \\ \text { Reference } & 14\end{array}$ 


\begin{abstract}
Research and Design of a Sample Heater for Beam Line 6-2c Transmission X-ray Microscope. VERONICA POLICHT (Loyola University, Chicago, Chicago, IL 60660) JOHANNA NELSON (Stanford Synchrotron Radiation Lightsource, SLAC National Accelerator Laboratory, Stanford, CA 94025)

There exists a need for environmental control of samples to be imaged by the Transmission X-Ray Microscope (TXM) at the SSRLs Beam Line 6-2c. In order to observe heat-driven chemical or morphological changes that normally occur in situ, microscopes require an additional component that effectively heats a given sample without heating any of the microscope elements. The confinement of the heat and other concerns about the heaters integrity limit which type of heater is appropriate for the TXM. The bulk of this research project entails researching different heating methods used previously in microscopes, but also in other industrial applications, with the goal of determining the best-fitting method, and finally in designing a preliminary sample heater.
\end{abstract}




\section{INTRODUCTION}

\section{Facilities}

SLAC National Accelerator Laboratory is one of 13 facilities in the United States equipped with a permanent source of synchrotron x-rays. Synchrotron radiation is produced at SLAC in the Stanford Synchrotron Radiation Lightsource (SSRL) by an electron beam subject to magnetic manipulation along a curved path. The bending of the electron beam's path results in a change in the beam's momentum and the emission of synchrotron x-rays which can be used to probe structures on the molecular level. The SSRL is equipped with facilities for several types of characterization techniques including x-ray diffraction, x-ray absorption spectroscopy, and macromolecular crystallography. Another type of technique which utilizes synchrotron x-rays is Transmission X-ray Microscopy (TXM). Transmission X-ray Microscopy is used to produce images of the sample in a way similar to Transmission Electron Microscopy (TEM), but with several marked advantages.

Beam Line 6-2c at the SSRL consists of a TXM, which utilized intensified synchrotron x-rays from an electron beam fed through a 54 pole wiggler. A beam of synchrotron x-rays builds as the electron beam travels sinusoidally through the wiggler's magnets of alternating polarity. Transmission X-ray Microscopy is a full field microscope, similar to a visible light microscope or TEM, that functions by training a focused, monochromatic beam of x-rays onto a sample where the rays are either absorbed or transmitted. Data is collected from the transmitted rays by a detector placed downstream of an objective lens. The TXM at Beam Line 6-2c can be characterized by the high image resolution of $30 \mathrm{~nm}$, large depth of field (20 - $60 \mu \mathrm{m})$, and a variety of imaging techniques. Unlike TEM, the high penetration depth of the synchrotron x-rays allows thick samples to remain largely intact and make it possible to image a complete structure. The x-rays range in energy from 5 - $14 \mathrm{keV}$ and by changing 
the energy of the x-ray beam incident on the sample, information can be gathered about the chemical structure of a sample. An advantage of using TXM for imaging is the ability to easily construct 3D tomography of a sample by collecting $2 \mathrm{D}$ images at many angles. The TXM uses hard x-rays to image a wide variety of samples including geological samples, lithium-ion batteries, and biological samples [1].

There is very limited available space around the sample stage of the TXM on Beam Line 6-2c. Between the pinhole, where the x-ray beam exits, and the zone plates downstream of the sample is roughly 1 inch of space for the sample and any additional components, as depicted in Fig. 1.

\section{Introduction of Problem}

To gain a better understanding of a sample's morphology and behavior, some samples require in situ conditions be maintained during imaging. Certain molecular and chemical reactions only occur at pressures and temperatures outside of those present in the open air of the TXM hutch and therefore need to be placed in an artificial reaction environment with an additional microscope component. The focus of this project has been to develop a heating device to be used at Beam Line 6-2c for the samples previously alluded to as well as other potential sample types.

The important characteristics of a heating device are as follows: it delivers well controlled, confined and measurable heat within a given temperature range and is capable of maintaining a given temperature for several hours as data is gathered. The heater will ideally perform between $50-500{ }^{\circ} \mathrm{C}$. Because the Beam Line equipment is also used by other users, the design of this heater must be easily installed and removed. Additionally, the heater device must physically be able to fit in the sample space of the TXM. A final consideration is the adaptability of the heater for different sample types; the two most general sample 
formats used in the TXM are either flat samples or samples contained within a capillary. An example of a heaters applicability is the real-time imaging of thermally driven reactions or the observation of a samples behavior and character across a temperature spectrum. For example, a recently proposed project for use of SSRL facilities would require their samples be kept at a temperature between 65 and $80{ }^{\circ} \mathrm{C}$. The proposers wish to conduct TXM and X-ray Absorption Near Edge Structure (XANES) imaging in order to study the nucleation and growth of a $\mathrm{ZnO}$ crystal film [2]. XANES is used to better understand chemical composition by examining the absorption of x-rays below, throught, and aboce the x-ray energy necessary to excite a core electron of a specific element.

There are many uses for heating devices in lightsource science and the ability to heat a sample adds another dimension to our understanding of elemental behavior over a spectrum of temperatures. Previously, the use of sample heaters in visible light and electron beam microscopes has successfully imaged the performance of materials at elevated temperatures. Therefore, by examining these heating devices, I plan to gather information for the design of a similar heating device to be used for x-ray light based equipment. The heating methods under consideration in this paper are three types of microelectromechanical resistive heating and two types of infrared heating: silicon wafer-based resistive, Kapton-based resistive, ceramic-based resistive, laser-based and infrared-based.

\section{Introduction to Sample Heaters}

A heater system is composed of the heating apparatus and a measurement apparatus. The two general methods of heating we have considered for use in our sample heater are infrared radiation (IR) and resistive heating methods. Infrared heat sources can be either coherent like lasers or incoherent like heat lamps. Infrared radiation trained onto the sample face is partially reflected and partially absorbed, resulting in sample heating. Heat lamps 
are radiative heaters, which consist of a heating element such as a metal filament that upon application of a large current radiates infrared light. Resistive heaters function by running a current through a metal wire with some internal resistance such that internal collisions of the moving electrons generate heat. This heat dissipates over the surface of the metal electrode and through the air. These general methods for heating function based upon Joule heating that running a current through a resistor will result in heat: $Q \propto I^{2} R$, where $Q$ is heat, $I$ is current, and $R$ is resistance. Resistance is a characteristic quality of a material and depends upon cross sectional area. Note that heat is only proportional to the right side of the equation.

There are several methods by which the temperature of a heater can be determined. Those considered in this project are direct measurement with a thermocouple, calibration of the heater before use, and calculating the local electrical resistance. All three of these methods are based upon electronic principles and take material properties into account. Calculating the temperature of a resistive heater requires information about the metal's resistance and the current as $Q$ is proportional to power, $\mathrm{P}: Q \propto P$. A thermocouple functions by measuring the current gradient across a junction of two different types of metal. By knowing the resistance of the respective metals you can calculate the temperature at the junction with the information about the current. There are few methods of heater calibration including using a heater on a test sample which has very well understood transitional properties. By comparing the well known materials transition states, such as melting, with the associated voltage and current applied to the heater you can calibrate the heater's resulting temperature. 
For the heating methods which require radiation to travel through the air, the Planck radiation function effectively describes emitted power per unit area of the emitting element:

$$
I=\frac{2 h c^{2}}{\left(e^{\frac{h c}{\lambda k T}}-1\right) \lambda^{5}}
$$

where $I$ is the energy per unit time per unit surface area, $\lambda$ is the wavelength of the emitted light, $T$ is the temperature of a black body, $h$ is the Planck constant, $c$ is the speed of light, and $k$ is the Boltzmann constant.

\section{METHODS}

My portion of this project consisted of investigating and working on a preliminary design for a heating device that would ideally adhere to all the previously stated parameters of removability and the constraints of its heating profile. After researching different methods of heating, I constructed a decision matrix. From this decision matrix I have deduced the best heat source options for Beam Line 6-2c. At this point I designed a preliminary heating apparatus, but due to time constraints did not see through to the beginning stages of fabrication.

The method by which research was conducted began with searching for any academic papers that used a given heating method. The papers that contained pertinent information were collected and used for reference material for constructing the decision matrix. Papers were either found by conducting Internet searches using Google Scholar, ArXiv, or the Stanford academic journal search engine $[3,4,5]$. In some instances a certain paper was found in another paper. Where there were no academic papers available relating to a certain heating method, information was gathered on the heating device from textbooks, manufacturer websites, and speaking with manufacturers' engineers. Textbook resources were discovered 
through Google Books and the manufacturer websites were found through Google search [6].

Based upon the gathered information, I then constructed a decision matrix; the decision matrix compares and ranks each heating method based upon how well it meets the previously determined desirable characteristics. Each of these characteristics has been given a weight 1 through 3, where a weight of 3 denotes more importance than a weight of 1 . Finally, the ranks given to the heaters are multiplied by the characteristic's weight, all of which are then summed. The heating method that has the lowest weighted rank is determined to be the best fit for our needs [Tables $3 \& 4$ ].

\section{RESULTS}

\section{Infrared Heaters}

\section{Infrared Heat Lamp Heaters}

Infrared heat lamp heaters are on the whole very effective at heating large areas with high intensity. The heat emitted by these lamps depends upon their color temperature, the temperature at which a black body would radiate with the same color. There exists commercially available infrared heating filaments, which operate within the desired temperature range decided upon for our sample heater. The main challenge of designing an infrared heat lamp heater for use in the TXM is managing to confine the heat to the sample face. As Fig. 1 shows, the components of the TXM near the sample stage are very close to where the desired heat would be. In order to preserve the integrity of these TXM elements we must ensure the elimination of all stray heat from the sample. To combat this problem we could use a small IR heat filament and create an apparatus such that the beam of infrared radiation would be focused and manipulated onto the sample. A preliminary design for such a heating apparatus is pictured in Fig. 2, and features the use of a reflecting mirror to direct 
the beam and a Cassegrain reflector to focus the beam to a small spot size. The use of a Cassegrain reflector is based upon the design for a Fourier Transform Infrared Spectrometer [7]. Another way to confine the IR heat would be implementing radiation-containment chamber made of ceramic.

Overall using an infrared heat lamp would be relatively costly in terms of purchasing components and the labor that would be required to build a viable heater. An infrared heat lamp would be difficult to use especially when the sample stage must be moved during imaging. A typical heat lamp alone costs around $\$ 200$. A recommendation I would suggest for making such a system easier to use would be placing the reflecting mirror and Cassegrain reflector on a mechanical stage, which could be controlled by computer. In this way, the two moveable components of the heater could be configured to move in sync with the sample stage. A final aspect to consider is the trade-off between filament size and the emissivity of the filament. Emissivity a is dimensionless quantity that relates a material's radiation of energy to the energy emitted by a black body at the same temperature. Emissivity depends on several factors including the thickness of a material; thinner materials will have a reduced emissivity.

\section{Laser Heaters}

Lasers are reliable, coherent heat sources; due to their coherence they can be manipulated in terms of intensity, beam size, polarization, and direction fairly easily. The use of an infrared laser to heat samples has been previously used in an x-ray setting in laser-heated diamond anvil cells $[8,9]$. These cells simultaneously pressurize and heat a sample and are able to reach temperatures above $1200{ }^{\circ} \mathrm{C}$. In these instances, a double-sided laser technique is implemented using Nd:YLF lasers. The use of two different lasers allows for minimal axial temperature gradient on the sample face. This is done by choosing to have the lasers 
function in complementary modes, one in Gaussian and the other doughnut-shaped modes. As a result, the axial temperature gradient is approximately $30{ }^{\circ} \mathrm{C}$, whereas a similar singlelaser system has an axial temperature gradient of hundreds of degrees. Another positive aspect of double-sided heating is its level of precision; this system implements several optical devices to obtain a heating area of $10-25 \mu \mathrm{m}[9]$.

The drawback of having the double-laser system is that the apparatus, pictured in Fig. 3, requires a lot of space and the entire set-up would be very expensive. A laser similar to those recommended, Diode Pumped Infrared $1030 \mathrm{~nm}$ CrystaLaser, is $\$ 7,600$, including its power supply [10]. A third reason this method is a poor fit is that the fabrication is too complicated for the scope of this project. This is a very precise and controlled heating method though, so I would recommend it in the case where someone could be dedicated to the design and fabrication of this specific apparatus.

\section{Resistive Heaters}

Resistive heaters include a broad range of heaters which most generally are composed of a base substrate with a metal electrode either embedded in or deposited on top of the base. Some resistive heaters have additional layers of insulation and electrodes placed on top of the two previously mentioned most basic layers. The heat character, dimensions, and physical character depend mostly upon the materials used for the base substrate and the metal electrode. For this reason, I have addressed three common types of resistive heaters; silicon-based, Kapton-based, and ceramic-based; separately. Figure 4 shows 3 examples of each of the three previously mentioned kinds of resistive heaters. Between the three types of resistive heaters the main areas of disagreement are the 1) operational temperature range, 2) cost, 3) manufacturing process difficulty, and 4) sample applicability. 


\section{Micro-Hotplate/Microelectromechanical System (MEMS) Heaters}

Micro-hotplates and MEMS are fabricated using a silicon wafer as their base substrate forming a frame and acting as a heat sink. Upon a silicon frame there is a membrane, which forms a window for radiation to penetrate. These heaters have been used as sample heaters in TEM systems because of their relative strength and thermal properties. Silicon has a high heat capacity and is strong at thicknesses above $50 \mathrm{~nm}$, but is brittle at thicknesses less than $50 \mathrm{~nm}$. Because of silicon's high heat capacity there is a large heat-drop off between the metal electrodes and the heater stand. The fabrication method of these siliconbased heaters has been performed and re-worked by many different research groups and as such these heaters have been developed to function in a broad temperature range. MEMS and micro-hotplates are able to operate at a wide range of temperatures with a highest mentioned operating temperature of $800{ }^{\circ} \mathrm{C}$ and the average operating temperature of 500 ${ }^{\circ} \mathrm{C}[11,12,13,14,15,16]$.

The silicon-based heaters perform well and offer a homogeneous heat distribution on the sample. Beckel, et al. specifically mentioned that the use of an integrated heater to anneal a crystallized film sample caused it to crack, but also that the annealing of said film took $15 \%$ of the time it took using other techniques [14]. Another paper tracked the drift of nanoparticles in their FOV and concluded that the heat from a spiraled Pt heater was well distributed. The findings are made based upon how consistent the drift rate of said particles was as it moved along a given path, giving a good measure of local heat gradient [17]. The use of resistive heating could effectively heat the sample at a steady, tunable, and readable temperature. Several previously used systems that require the use of a micro-hotplate have used either a heating element with multiple connections such that it can simultaneously be controlled and read, or a system with dedicated components for heating and for temperature measurement. 
Several of the papers that I have read have stated in various levels of detail the exact fabrication methods which indicate that this would be a difficult apparatus to pursue without outside help. These apparatuses are produced using several nanofabrication and chemical processes and would only be able to be performed in the cleanroom environment of an outside nanofabrication lab. Generally, fabrication employs several machining and microfabrication techniques. A few examples of the type of processes are lift-off technique for the deposition of the Pt heating element and low-pressure chemical vapor deposition for the placement of a SiN membrane layer on top of the heating element $[11,12,13,14,15,16]$. Additionally, E.A. Olson, et al. details specific instructions on how to best choose your conducting material $[16]$.

There are also many manufacturers and distributors of silicon-based resistive heaters. Many of the companies that produce these also offer customization services to ensure that the heater meets the customers specifications. The trade off between fabricating the apparatus ourselves and buying one from an outside source is cost. One silicon-based micro-heater (10 mm x $5 \mathrm{~mm} \times 0.2 \mathrm{~mm}$, gold electrode, $350 \mathrm{~nm}$ thick) is $\$ 82.00$ without any customization; requesting custom dimensions increases the price [18]. Though one heater is not on it's own expensive, most companies have minimum order sizes and the outcome is a large, expensive order of heaters. Another drawback of using the silicon-based heaters is that it, because silicon is not very flexible, it would only apply homogeneous heat to flat samples. A final thing to consider is that, as already mentioned, the devices were designed for and used for TEM and SEM measurements. The use of the TEM and SEM places the micro-hotplate in a vacuum and as such we cannot assume that our observed heat drop-off will not be the same as what had been observed in their papers. That said, the spacing between the electrode patterns would be larger than the x-ray beam diameter and so, expecting that the heat will be homogeneous in that region is not a terrible assumption. 


\section{Kapton Heaters}

Kapton heaters use Kapton film as their base substrate. Kapton film is characterized by its excellent thermal and physical properties, which make it a good component for a prospective sample heater. Kapton film's limits have been well tested and so many of its thermal properties are known. Kapton has a thermal conductivity on the order of $10^{-1} \frac{W}{m K}$, which correlates to high insulative properties. For comparison, Table 1 lists several materials and their thermal conductivities [19].

There are several companies which specialize in the production of Kapton heaters. The majority of these companies advertise customizability in terms of dimensions of the heater and of the metal foil materials used for the metal electrode. The dimensions of the heater, such as thickness and distance between the electrodes, depend upon the metal material chosen.

The problem met with using a Kapton-based heater is the maximum operational temperature. Table 2 shows three Kapton-heater companies and the associated maximum temperatures attainable. These listed temperatures are well below what we had hoped for in a maximum heating temperature $\left(500{ }^{\circ} \mathrm{C}\right)$. Kapton film itself maintains its structural integrity up to temperatures as high as $400{ }^{\circ} \mathrm{C}$ [19]. This would indicate that the temperature limitations stem from the metal used for the electrode or the adhesive used in fabrication.

Overall I think that Kaptons physical properties would make it a good material for a heater. Kaptons flexibilty means it could homogeneously heat both planar surfaces and surfaces which are gently curved. However, as far as prefabricated heaters go those available will not operate in the temperature range we require. 


\section{Ceramic Heaters}

Ceramic materials are used as heater substrates because of their favorable physical and electrical properties including good strength, high temperature stability, low dielectric constant, and high electrical insulation. Ceramic infrared heaters are often used in industrial manufacturing as well as domestic and pet care applications. More so than silicon- and Kapton-based heaters, ceramic-based heaters are limited in terms of the dimensions at which they can be produced to function. Ceramic heaters can be manufactured in many shapes including squares, circles, and cylinders with the metal wires being embedded on the face for the square and circle and within the inner portion for the cylinder. This offers some flexibility in applicable samples as you could potentially use a square or circle for flat samples and a cylindrical for a capillary-bound sample. Unfortunately, the heat is not easily confined in the case of the square or circular heater with there being too much risk of the TXM elements surrounding the sample being affected by stray heat. This leaves the cylindrical heater, which is an excellent option for samples contained within a capillary.

There are several cylindrical ceramic based heaters available commercially and offer some level of customizability. A ceramic heater generally consists of a ceramic shell with a metal coil embedded in the wall of the shell. The wires are seldom left uncovered as they reach very high temperatures; clamshell furnace elements reaching temperatures greater than $1100{ }^{\circ} \mathrm{C}$ [20]. The metal wires are usually either covered completely with a thin layer of ceramic or are partially covered by ceramic bars.

Another option is fabricating a ceramic-based heater by threading a hollow cylinder of Macor machinable ceramic with a metal wire. Macor retains structural integrity up to $1000{ }^{\circ} \mathrm{C}$ and has similar chemical and thermal properties to ceramics used in the fabrication of commercial furnaces. A 1/2" diameter 3 long cylinder of Macor costs $\$ 32.00$ [21]. Fabrication 
of this type of heater would be relatively easy with the most difficult portion being threading the wire in the Macor. A preliminary design of a ceramic-based heater produced in-house at SLAC is pictured in Fig. 7.

\section{CONCLUSIONS}

Based upon the decision matrix in Tables 3 and 4, the best option for our sample heater is the Kapton-based resistive heater. While the Kapton heaters are not able to function through the entirety of our desired temperature range, the benefits in terms of size, cost, and ease of production make Kapton a good option for the current needs of Beam Line 6-2c. Because the Kapton heater only is applicable to flat or gently-curved surfaces and because it can only operate up to $200{ }^{\circ} \mathrm{C}$, we have chosen a second heating type to cover a wider sample range. The second heater type is the cylindrical ceramic-based heater. Ceramic heaters are relatively easy to produce, have several methods of measuring the temperature, and operate very well within the desired temperature range. The combination of these two heating methods covers most sample types for imaging in the TXM and covers the characteristics we desired in a sample heater. Figures 5 and 6 show SketchUp images of the proposed Kaptonbased sample heater to be used on flat and gently-curved sample types [22]. The heater is based upon a Kapton heater distributed by OMEGA Industries [23].

\section{ACKNOWLEDGMENTS}

I would like to thank the Department of Energy and the faculty at SLAC who have made this internship possible for myself and many other undergraduate students. I would like to thank Johanna Nelson for mentoring me throughout this project and for ensuring that I had a meaningful experience at SLAC. Finally, I'd like to thank the other SULI student interns who helped make this summer wonderful. 


\section{REFERENCES}

[1] J. Andrews, S. Brennan, C. Patty, K. Luening, P. Pianetta, E. Almeida, M. C. H. van der Meulen, M. Feser, J. Gelb, J. Rudati, A. Tkachuk, and W. B. Yun, "A high resolution, hard x-ray bio-imaging facility at ssrl," Synchrotron Radiation News, vol. 21, no. 3, p. 26, 2008.

[2] A. Cruickshank, "In-situ txm of the nucleation and growth of electrodeposited zno nanocrystals," June 2011, proposed Usage.

[3] Google Scholar Database. [Online]. Available: http://scholar.google.com/

[4] ArXiv: Open access Scholarly Database. [Online]. Available: http://arxiv.org/

[5] Stanford Research E-Library. [Online]. Available: http://www-group.slac.stanford. edu/library/

[6] Google Books Internet Database. [Online]. Available: http://books.google.com/

[7] S. Slobodan and Y. Ozaki, Eds., FT-IR Imaging Hardware. John Wiley and Sons, 2011, sections 3.1-3.2.

[8] Y. Meng, G. Shen, and H. K. Mao, "Double-sided laser heating system at hpcat for in situ x-ray diffraction at high pressures and high temperatures," Journal of Physics: Condensed Matter, vol. 18, no. 25, p. S1097, 2006. [Online]. Available: http://stacks.iop.org/0953-8984/18/i=25/a=S17

[9] G. Shen, M. L. Rivers, Y. Wang, and S. R. Sutton, "Laser heated diamond cell system at the advanced photon source for in situ x-ray measurements at high pressure and temperature," AIP, vol. 72, no. 2, pp. 1273-1282, 2001. 
[10] T. Zaki, August 2011, person correspondance with CrystaLaser technical engineer. [Online]. Available: www.crystalaser.com

[11] D. Briand, A. K. B. van der Schoot, U. Wiemar, N. Barsan, W. Gpel, and N. de Rooij, "Design and fabrication of high-temperature micro-hotplates for drop-coated gas sensors," Sensors Actuators B, vol. 68, pp. 223-233, 2000. [Online]. Available: www.elseview.com/locate/snb

[12] E. de Smit, I. S. J. F. Creemer, G. H. Hoveling, M. K. Gilles, T. Tyliszczak, P. J. Kooyman, H. W. Zandbergen, C. Morin, B. M. Weckhuuysen, and F. M. F. de Groot, "Nanoscale chemical imaging of a working catalyst by scanning transmission x-ray microscopy," Nature, vol. 456, pp. 222-226, 2008.

[13] M. Z. nd E.A. Olson, R. Twesten, J. Wen, L. Allen, I. Robertson, and I. Petrov, "In situ transmission electron microscopy studies enabled by microelectromechanical system technology," J. Mater. Res., vol. 20, no. 7, pp. 1802-1807, 2005.

[14] D. Beckel, D. Birand, A. Bieberle-Hütter, J. Courbat, N. F. de Rooij, and L. J. Gauckler, "Electrical conductivitiy of lscf thin films for sofc on micro hotplates," work performed by members of ETH Zürich and the University of Neuchatel.

[15] M. Jeule and L. Gauckler, "Miniaturised arrays of tin oxide gas sensors on single microhotplate substrates fabricated by micromolding in capillaries," Sensors and Actuators B, vol. 93, pp. 100-106, 2003. [Online]. Available: www.elseview.com/locate/snb

[16] E. A. Olsen, M. Y. Efremov, M. Zhang, Z. Zhang, and L. H. Allen, "The deisgn and operation of a mems differential scanning nanoncalorimeter for high-speed heat capacity measurements of ultrathin films," Journal of Microelectromechanical Systems, vol. 12, no. 3, pp. 355-364, June 2003. 
[17] J. F. Creemer, S. Helveg, G. H. Hoveling, S. Ullmann, A. M. Molenbroek, P. M. Sarro, and H. W. Zandbergen, "Atomic-scale electron microscopy at ambient pressure," Ultramicroscopy, vol. 108, pp. 993-998, April 2008.

[18] M. Cox and P. Anastasi, July 2011, person correspondance with Silson Ltd regarding the purchasing of a micro-hotplate. [Online]. Available: http://www.silson.com/

[19] Summary of Properties for Kapton Polyimide Films, DuPont. [Online]. Available: http://www2.dupont.com/Kapton/en_US/assets/downloads/pdf/summaryofprop.pdf

[20] The Mellen Company; produce ceramic cylindrical furnaces and heaters. [Online]. Available: http://www.mellencompany.com/

[21] Astro Met, Inc.; distributor of Macor glass ceramic produced by Corning Incorporated. [Online]. Available: http://www.astromet.com/macor.htm

[22] Free 3D Modeling Software by Google. [Online]. Available: http://sketchup.google.com/

[23] OMEGA; produce Kapton Flexible Heaters. [Online]. Available: http://www.omega. $\mathrm{com} /$

[24] G. J. Nelson, W. M. Harris, J. J. R. Izzo, K. N. Grew, W. K. S. Chiu, Y. S. Chu, J. Yi, J. C. Andrews, Y. Liu, and P. Pianetta, "Three-dimensional mapping of nickel oxidation states using full field x-ray absorption near edge structure nanotomography," AIP, vol. 98, no. 17, p. 173109, 2011.

[25] Newport Corporation; produce IR Heating Elements. [Online]. Available: http: //newport.com/ 


\section{FIGURES}

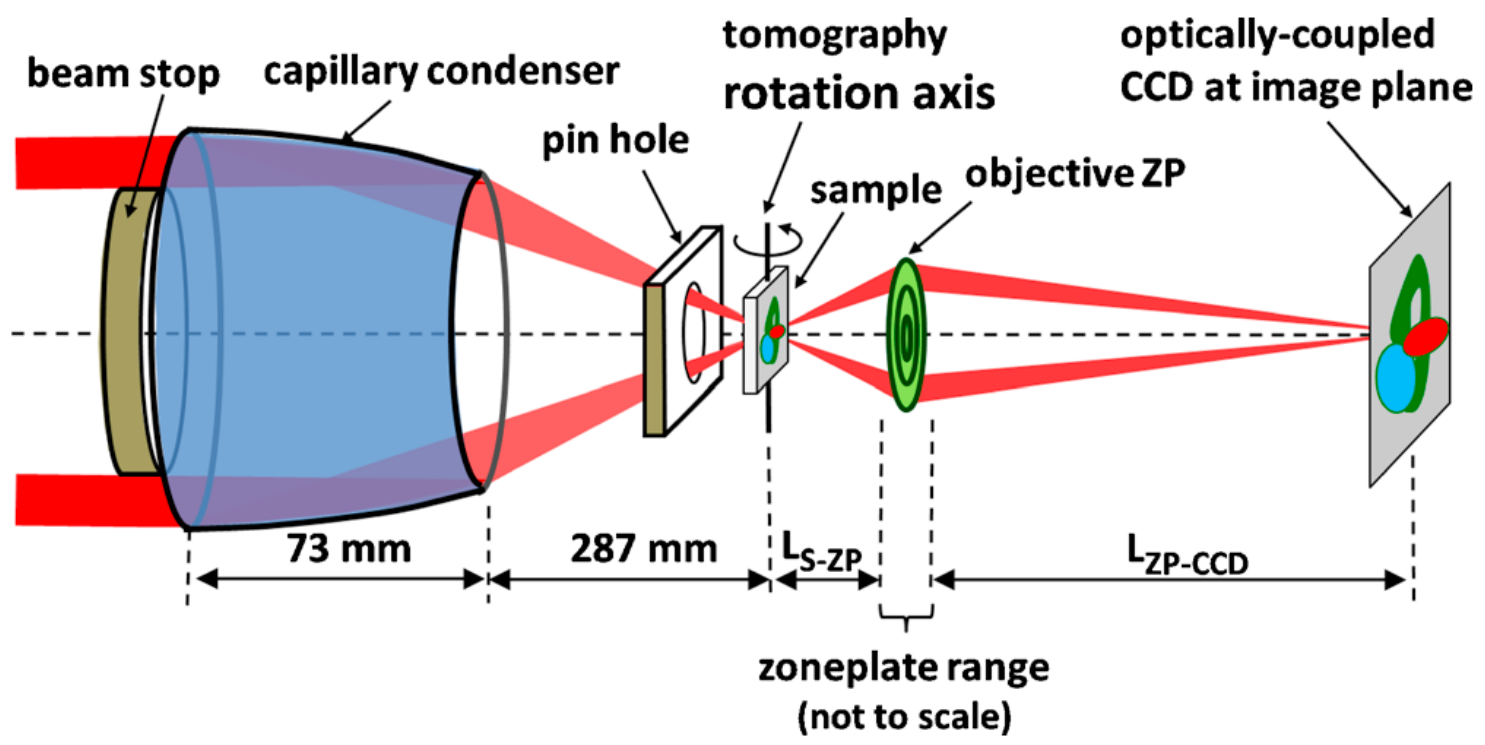

Figure 1: TXM schematic of the optics involved in full field nanotomography. The x-rays are focused by the capillary condenser and unfocused rays are obstructed by the pin hole and beam stop. The x-rays are focused onto the sample and the transmitted rays are transformed by the objective zone plate and are finally detected by an optically-coupled CCD. The sample is able to rotate about an axis in cases where tomography is desired. [24] 


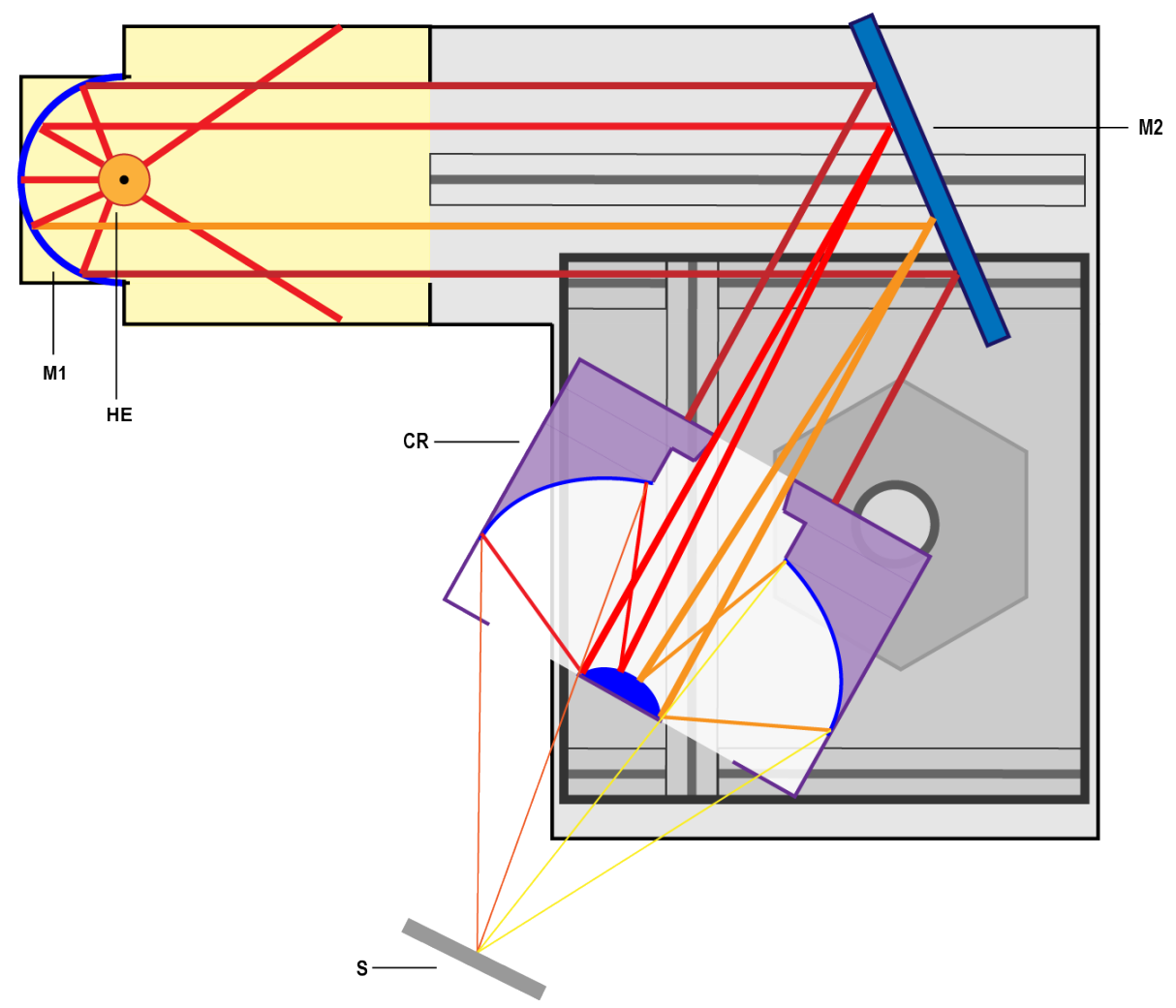

Figure 2: Schematic depicting possible IR Heat Lamp Apparatus. The Heat Emitter (HE) is backed by a primary reflecting mirror (M1) which directs the radiation towards a second reflecting mirror (M2) which is also acts as a directing mirror. The radiation is then reflected into a Cassegrain Reflector (CR) where it is focused onto the sample (S) face. Both M2 and $\mathrm{CR}$ are placed on mechanical tracks such that they are able to move as adjustments are needed. 


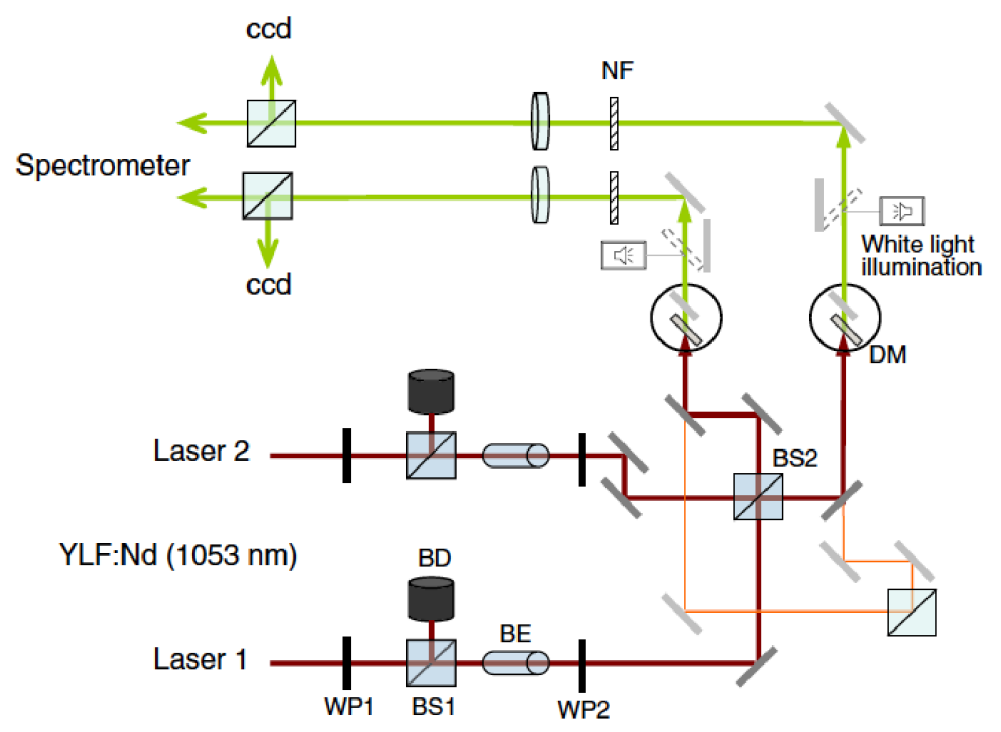

Figure 3: Schematic of the optics involved in manipulating and focusing the laser beams. In this diagram WP represent wave plate, BS represent beam splitter, BD represents beam dump, BE represents beam expander, DM represents dichroic mirror, ccd represents a CCD camera. WP1, BS1, and BD regulate the power of the laser beam. The beam is then combines and redistributed by WP2 and BS2. [8].
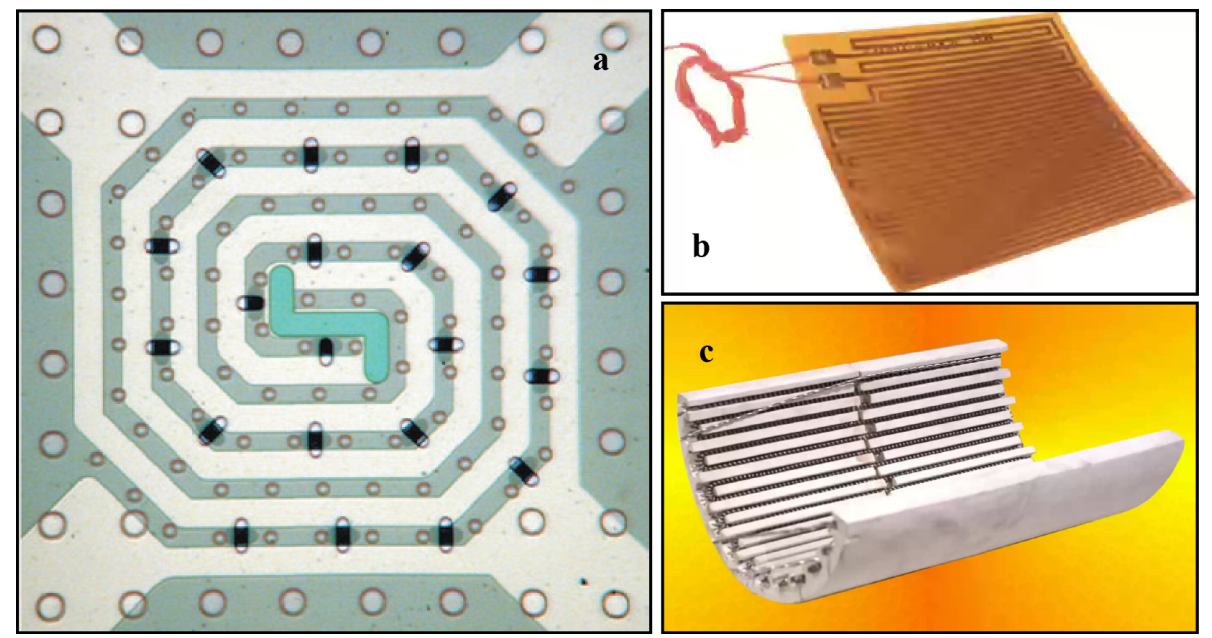

Figure 4: Depicted are various resistive heaters. 4a A silicon-based MEMS with spiraled Pt electrode pattern. [17] 4b A Kapton-based flexible heater with metal-foil meandering electrode pattern and two leads. [23]. 4c A ceramic-based heater where the ceramic halfcylinder is embedded with metal wire, which is partially obscured with additional ceramic bars to keep wire from contacting sample. [20]. 


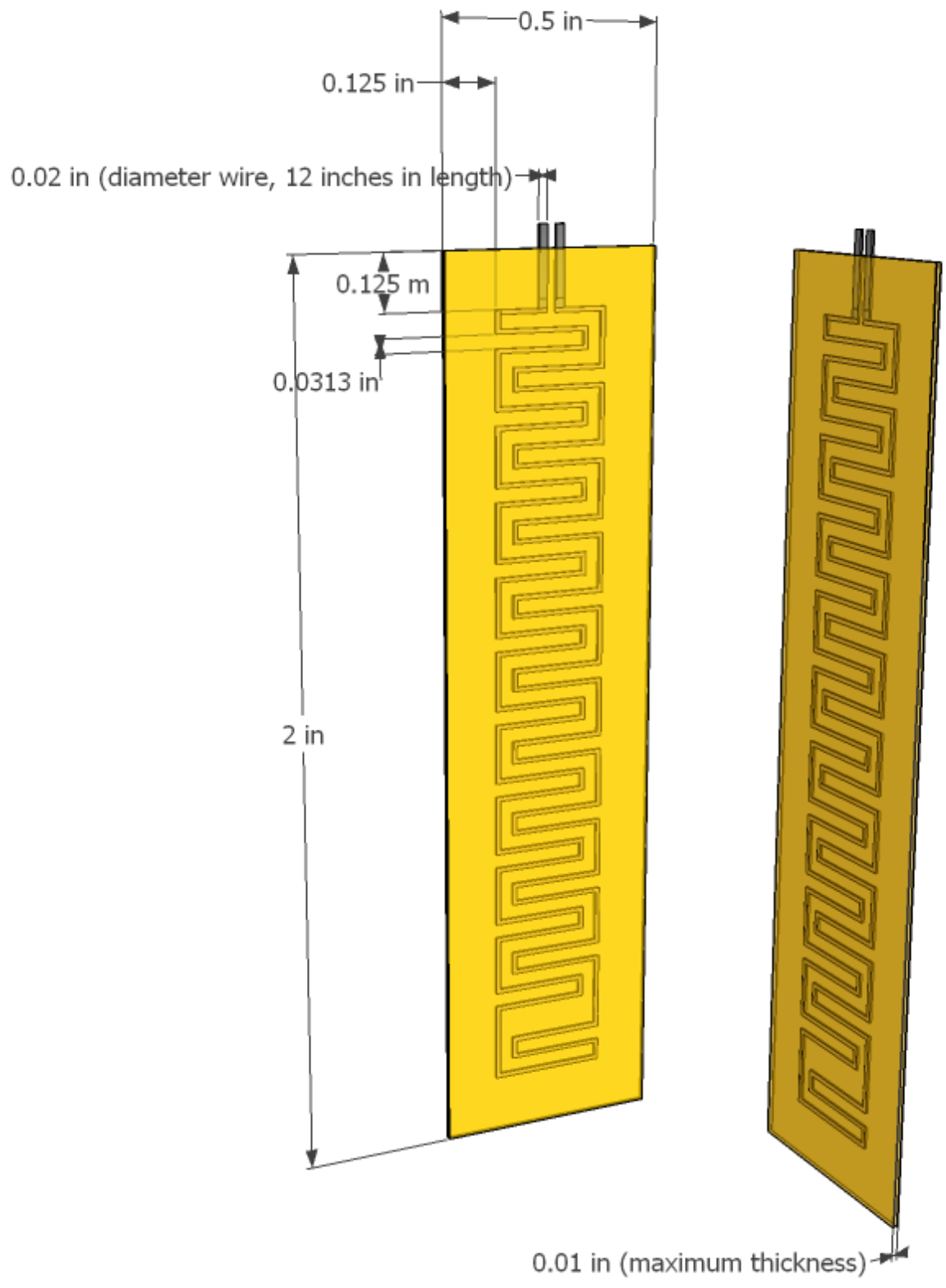

Figure 5: SketchUp image of the proposed Kapton Flexible Heater with two 12" FEP insulated 24 gauge wire leads. OMEGA Flexible Heater, $0.5 "$ x 2" ; spacing between etched foil electrodes $1 / 32$ "; from heater edge to electrode edge $1 / 8$ "; maximum heater thickness 0.01 " $[23]$. 


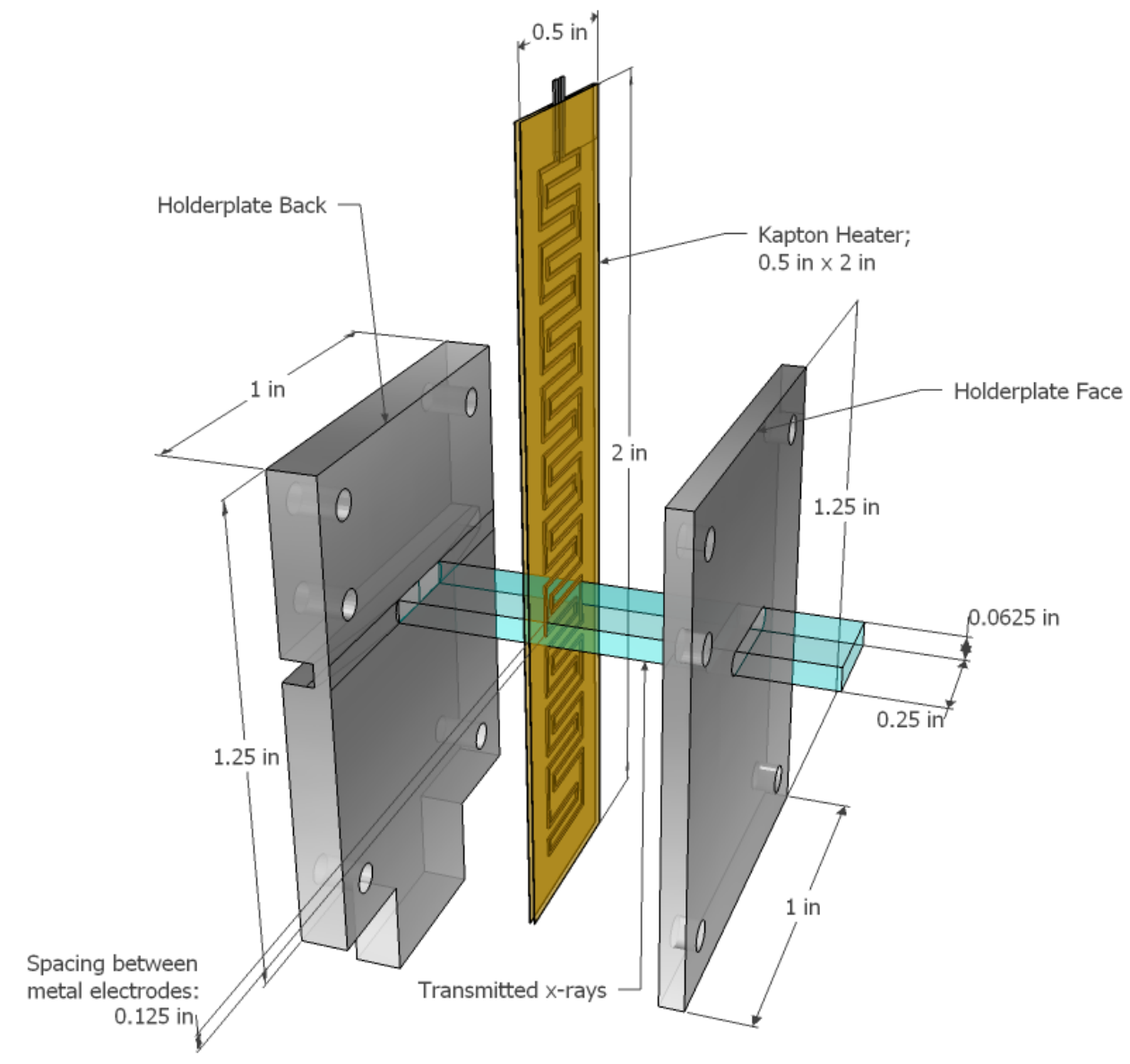

Figure 6: SketchUp image of the expanded sample holder. The proposed Kapton heater from Figure 5 is positioned between the front and back sample holderplates. Holderplates are made of aluminum and were designed by Johanna Nelson. A projection of the spatial range available for $\mathrm{x}$-rays to be transmitted shown in blue; note that the actual beam is on the order of $15-30 \mu \mathrm{m}$. The openings in the holderplates are $1 / 4 " \times 1 / 16 "$. 


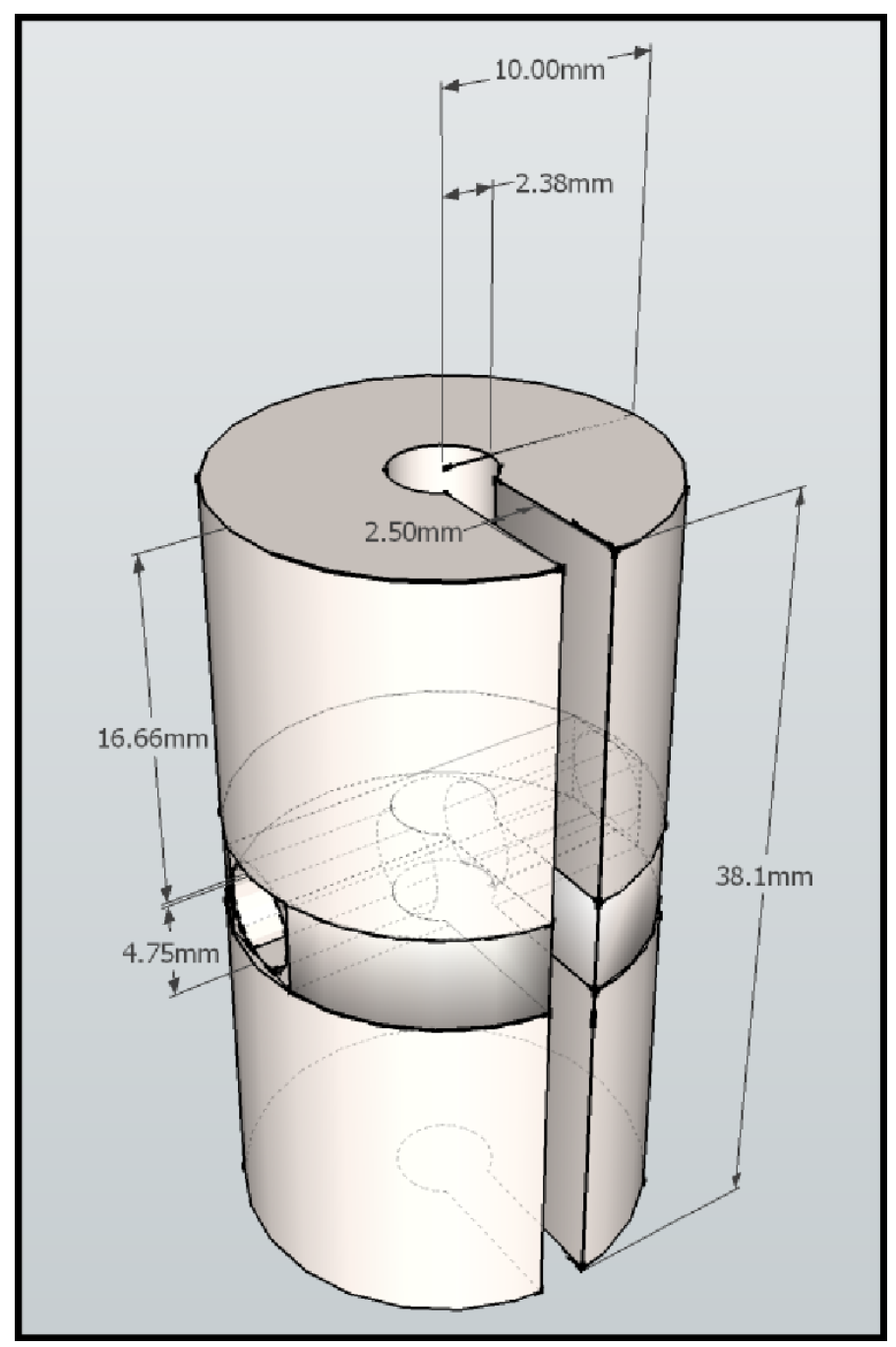

Figure 7: SketchUp image of a 3D model of a ceramic-based resistive heater that would be fabricated in-house using machinable Macor ceramic. The ideal design for such a ceramic heater would have the wire partially embedded within the ceramic. This design would feature a solid ceramic body with a vertical slit, which enables the user to slide the heater around a capillary. The ceramic cylinder would feature a circular, horizontal gap to allow x-ray transmittance through the sample. This design could be mounted externally to the table adjacent to the sample stage, allowing the sample to rotate for tomography while keeping the heater still. [21] 


\section{TABLES}

\begin{tabular}{|l|c|c|c|c|c|c|c|c|}
\hline \hline Material & Air & Wood & Kapton & Water & Glass & Macor Ceramic & Aluminum & Copper \\
\hline$k[\mathrm{~W} / \mathrm{mK}]$ & 0.025 & 0.04 & 0.37 & 0.6 & 1.1 & 1.46 & 237 & 401 \\
\hline
\end{tabular}

Table 1: Materials and their thermal conductivities $[\mathrm{W} /(\mathrm{mK})]$. Kapton polyimide films thermal conductivity than glass and ceramic but is larger than air and wood; its thermal conductivity is one thousandth of copper.

\begin{tabular}{|l|c|c|c|c|c|}
\hline \hline Company & $\begin{array}{c}\text { Operating } \\
\text { Temp. Extrema } \\
\text { of the Kapton } \\
\text { Heating }\left({ }^{\circ} \mathrm{C}\right)\end{array}$ & $\begin{array}{c}\text { Heater Thickness } \\
(\mathrm{nm})\end{array}$ & $\begin{array}{c}\text { Spacing } \\
\text { Between } \\
\text { Electrodes } \\
(\mathrm{mm})\end{array}$ & $\begin{array}{c}\text { Minimum } \\
\text { Dimensions } \\
(\mathrm{mm} \times \\
\mathrm{mm}\end{array}$ & $\begin{array}{c}\text { Cost per } \\
\text { Heating } \\
\text { Unit }^{\dagger}\end{array}$ \\
\hline $\begin{array}{l}\text { Durex } \\
\text { Industries }\end{array}$ & $\begin{array}{c}\text { Low -195, } \\
\text { High: 200 }\end{array}$ & $\begin{array}{c}0.1524-0.3048 ; \\
\text { Standard: } \mathbf{0 . 1 7 8}\end{array}$ & & $25.4 \times 25.4 /$ & \\
\hline OMEGA & $-200, \mathbf{2 0 0}$ & $\mathbf{0 . 2 0 3}$ & 0.79 & $6.35 \times 6.35 /$ & $\$ 31$ \\
\hline $\begin{array}{l}\text { Birk Design } \\
\text { and } \\
\text { Manufacturing }\end{array}$ & $\mathrm{N} / \mathrm{A}, \mathbf{1 2 0 / 2 6 0 ^ { \ddagger }}$ & $0.07-0.254$ & & $12.7 \times 12.7 /$ & \\
\hline
\end{tabular}

Table 2: Companies which produce customizable Kapton-based resistive heaters and their respective characteristics and dimensions. ${ }^{\dagger 5} \mathrm{~W} / \mathrm{in}^{2}, 0.5 ”$ x $2 "$; ${ }^{\ddagger}$ Acrylic bonded Kapton has max. capability of 120 


\begin{tabular}{|c|c|c|c|c|c|c|c|c|c|c|}
\hline 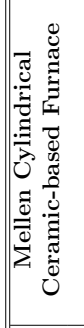 & 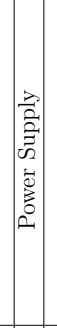 & 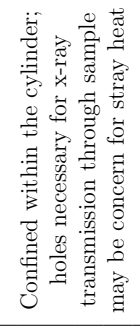 & 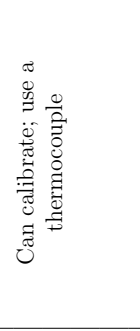 & 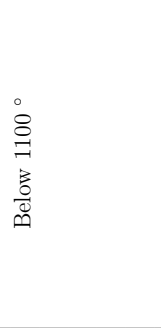 & 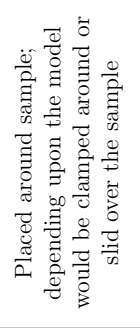 & 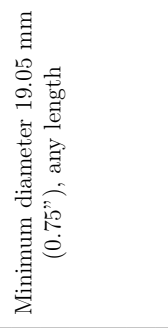 & 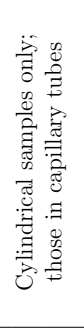 & $\frac{\varangle}{z}$ & 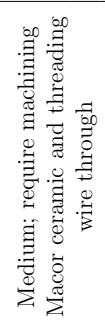 & 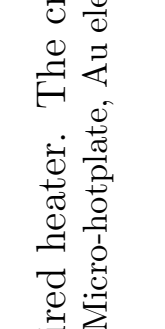 \\
\hline 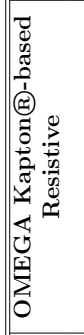 & 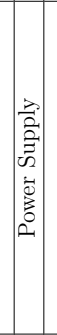 & 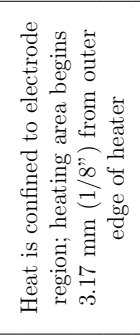 & 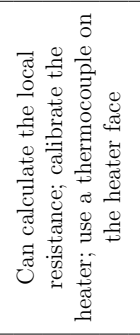 & 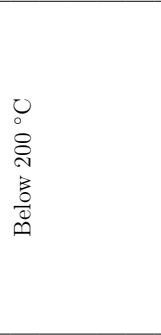 & 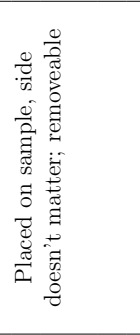 & 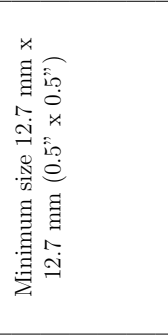 & 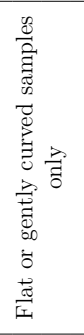 & $\begin{array}{l}\vec{y} \\
\stackrel{8}{1} \\
\vec{B}\end{array}$ & 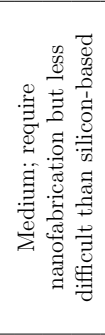 & 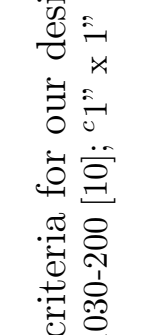 \\
\hline 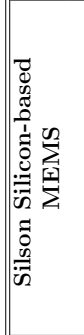 & 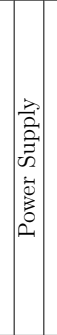 & 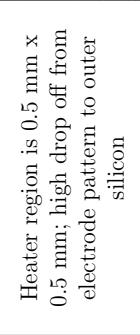 & 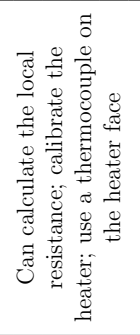 & 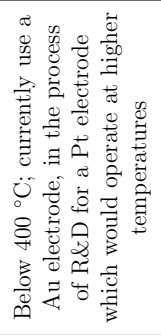 & 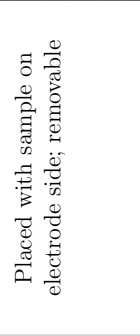 & 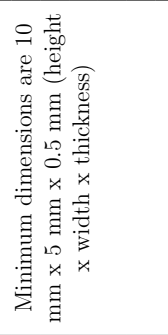 & 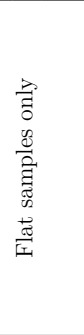 & $\underset{\substack{\vec{\infty} \\
\leftrightarrow}}{\stackrel{f}{\vec{\infty}}}$ & 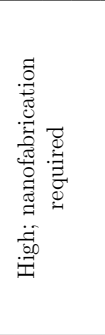 & 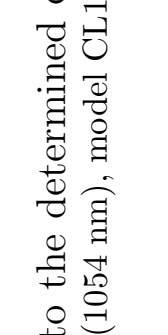 \\
\hline 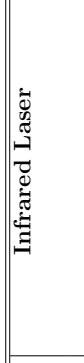 & 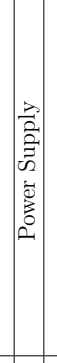 & 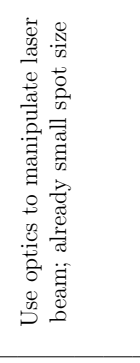 & 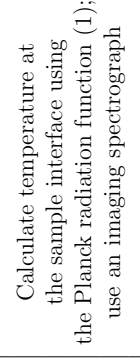 & 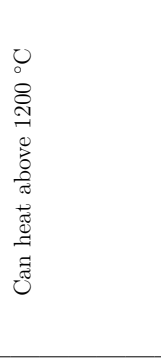 & 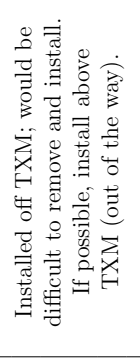 & 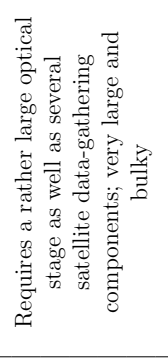 & 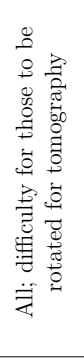 & 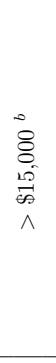 & 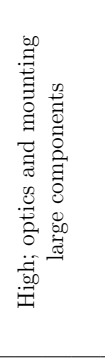 & 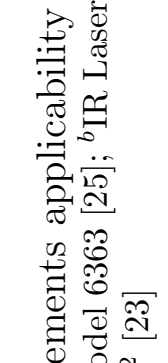 \\
\hline 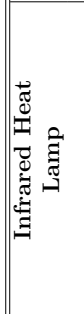 & 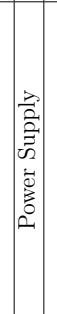 & 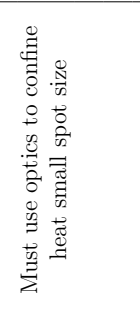 & 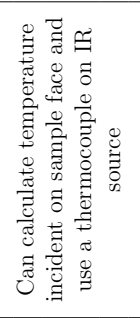 & 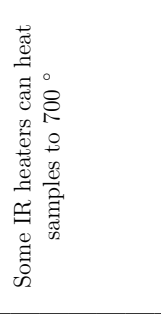 & 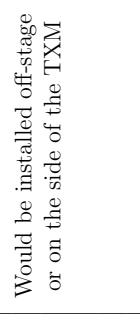 & 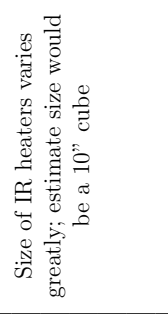 & 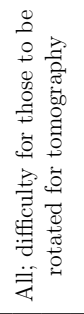 & 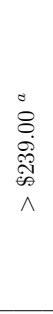 & 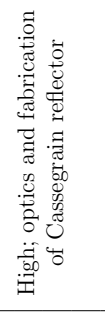 & 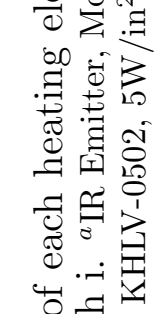 \\
\hline & 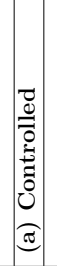 & 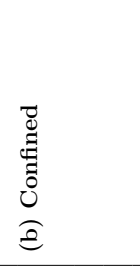 & 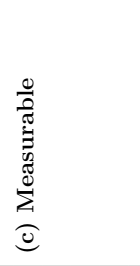 & 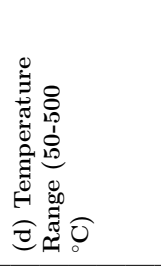 & 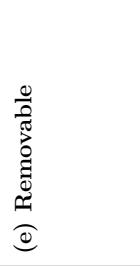 & 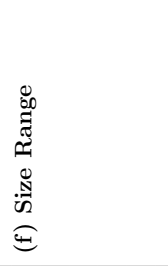 & 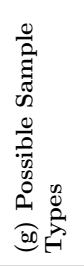 & $\begin{array}{l}\vec{b} \\
\hat{d} \\
\hat{\Xi}\end{array}$ & 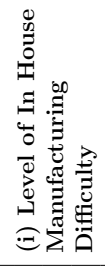 & 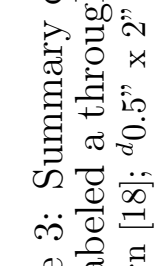 \\
\hline
\end{tabular}




\begin{tabular}{|l|c|c|c|c|c|c|c|c|c|c|}
\hline \hline & $\mathrm{a}$ & $\mathrm{b}$ & $\mathrm{c}$ & $\mathrm{d}$ & $\mathrm{e}$ & $\mathrm{f}$ & $\mathrm{g}$ & $\mathrm{h}$ & $\mathrm{i}$ & \\
\hline Weight & 1 & 2 & 2 & 3 & 1 & 3 & 1 & 3 & 3 & \\
\hline \multicolumn{8}{|c|}{ Rank (1-best, 5-worst) } \\
\hline Heat Lamp & 1 & 5 & 3 & 5 & 4 & 4 & 3 & 3 & 4 & Weighted Rank \\
\hline Laser & 1 & 1 & 1 & 1 & 5 & 5 & 1 & 5 & 5 & 59 \\
\hline Silicon & 1 & 2 & 4 & 2 & 1 & 1 & 5 & 2 & 3 & 43 \\
\hline Kapton & 1 & 3 & 5 & 2 & 1 & 2 & 2 & 1 & 2 & $\mathbf{4 1}$ \\
\hline Ceramic & 1 & 4 & 1 & 4 & 3 & 3 & 5 & & 1 & $\mathbf{4 3}$ \\
\hline
\end{tabular}

Table 4: Each of the criteria a through i were weighted, as shown. Then the heating methods were ranked for each of the criteria, 1 being the best and 5 the worst. The total indicates the sum of the heaters rank for a respective criteria multiplied by that criterias weight. The best scores are the lowest. 